\title{
Design Preserving Garment Transfer
}

\author{
Remi Brouet ${ }^{13} \quad$ Alla Sheffer $^{2} \quad$ Laurence Boissieux $^{3} \quad$ Marie-Paule Cani $^{13}$ \\ ${ }^{1}$ Grenoble University $\quad{ }^{2}$ University of British Columbia $\quad{ }^{3}$ INRIA
}
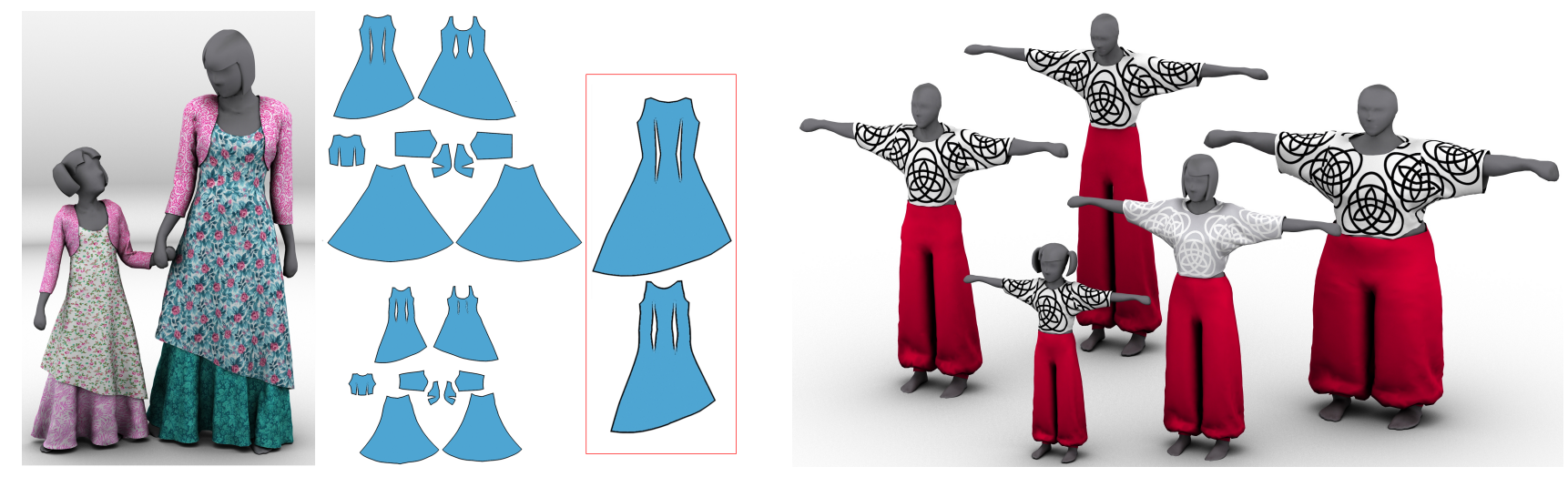

Figure 1: Design preserving garment transfer: (left) transfer of a multi-layer outfit from a woman to a young girl; automatically graded patterns (bottom) shown to scale. The zoomed-in source and target patterns for the back panel highlight the subtle changes in shape. (right) Dancer outfit transferred from the female in the center to a variety of other characters. (c)Laurence Boissieux 2012

\section{Abstract}

We present a fully automatic method for design-preserving transfer of garments between characters with different body shapes. For real-life garments, such transfer is performed through a knowledge intensive and time consuming process, known as pattern grading. Our first contribution is to reformulate the criteria used in professional pattern-grading as a set of geometric requirements, respectively expressing shape or design preservation, proportionality, and fit. We then propose a fully automatic garment transfer algorithm which satisfies all of these criteria while ensuring the physical plausibility of the result. Specifically, we formulate garment transfer as a constrained optimization problem and solve it efficiently through iterative quadratic minimization. As demonstrated by our results, our method is able to automatically generate design-preserving versions of existing garments for target characters whose proportions and body shape significantly differ from those of the source. The method correctly handles the transfer of multiple layers of garment. Lastly, when source 2D patterns are available, we output graded patterns suitable for manufacturing the transferred garments. Our fully automatic design-preserving transfer method leads to significant time savings for both computer artists and fashion designers.

CR Categories: I.3.7 [Computer Graphics]: Three-Dimensional Graphics and Realism-;

Keywords: Garment modeling, geometry processing, transfer methods, resizing

Links: DL $₫$ PDF

\section{Introduction}

Modeling of complex real or virtual garments is a highly time consuming and knowledge intensive task. For instance, it took an experienced computer artist three hours to generate the woman's outfit in Figure 1 (left), a process that included creating 2D patterns, placing them on the mannequin, running cloth simulation and then iteratively adjusting patterns and parameters, repeating the process until the desired effect was achieved. If we wished to design a similar looking outfit for a young girl who has very different proportions, see Figure 1 (left), this tedious process would need to be repeated practically from scratch. Consequently, dressing a large number of virtual characters in similarly designed outfits, Figure 1 (right), is currently prohibitively time consuming, even if state-of-the-art garment modeling interfaces such as [Umetani et al. 2011] are used.

In real-life, professional pattern makers adapt garments to people with different proportions and body-types through a complex process known as grading or pattern grading. Manual grading aims to preserve the design or look of the garment while adjusting it to fit the proportions of the new wearer [Moore et al. 2001]. This process requires significant specialized expertise and relies on a set of precomputed rule tables for standard body sizes. Applying a similar process to virtual garments can be especially challenging since the characters to be dressed often have non-standard body proportions for which no rule-tables exist. While skinning-type garment transfer techniques [Cordier et al. 2003; Wang et al. 2005] adjust the garment to fit the target character's proportions reasonably well, they often fail to preserve the original design, requiring manual editing to recover it [Meng et al. 2012].

Instead, we focus on automatic design preserving garment grading or transfer, introducing a method that produces garments that fit the proportions of the new wearer while retaining the original design as much as possible. As emphasized in the literature, garment transfer is an ill-posed problem. Formalizing what makes a 

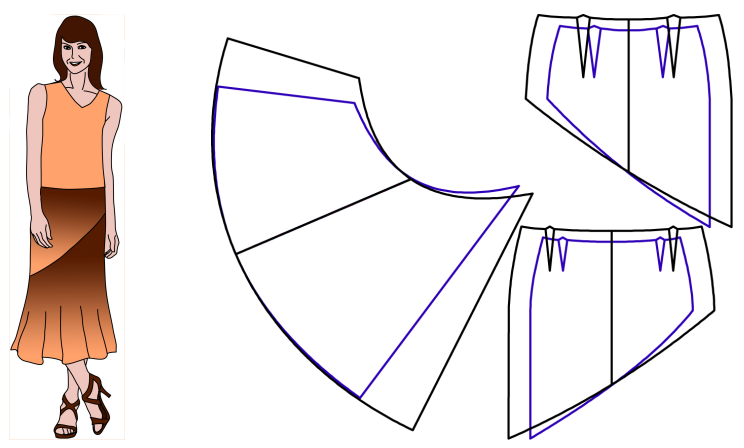

Figure 2: Traditional patterns for a skirt for small and large sizes (C)LaurenceBoissieux 2012).

garment look the same on characters of different body shapes, although the input and output garment surfaces obviously differ, is in itself a challenge. Therefore, our first contribution, presented in Section 3, is to analyze professional pattern-grading criteria and reexpress them in terms of geometric requirements for garment-mesh transfer. We then introduce a garment transfer algorithm that satisfies these requirements, as our main contribution. We formulate garment transfer as a constrained optimization problem which optimizes design preservation subject to a number of geometric constraints arising from proportionality, plausibility and other considerations elaborated upon in Section 3. To efficiently generate the graded garments we solve this problem using an iterative process, where each iteration consists of solving a quadratic minimization problem.

As demonstrated by the results, our method enables fully automatic, realistic, design preserving transfer of garments between characters with vastly different body shapes and proportions. Consequently, it significantly simplifies the modeling of new dressed characters pre-existing wardrobes can be instantly transferred from one character to another without the need for any specific user skill. Our method also allows efficient generation of large groups of similarlydressed characters for the film industry - e.g. warriors, workers, dancers, etc, without the usual restriction to clone-like models.

Plausible virtual garments have a low distortion mapping to a set of 2D patterns, enabling texture mapping and actual manufacturing. Our design preservation criteria, as discussed in Section 3 explicitly aim to preserve plausibility. Thus given the $2 \mathrm{D}$ patterns for the source garments, our method directly generates the $2 \mathrm{D}$ patterns for the transferred one (Figure 1, middle).

\section{Related work}

Previous research relevant to our work can be roughly split into three areas: general garment modeling approaches, grading and transfer methods for garments, as well as resizing and transfer methods for other domains.

Garment modeling The traditional process for virtual garment modeling [Protopsaltou and Luible 2002] begins with the design of $2 \mathrm{D}$ patterns. These patterns are then stitched together and lifted to $3 \mathrm{D}$, where a physics-based simulation is used to generate the final 3D shape. The process is hard to control, and needs to be iterated until the designer is satisfied with the results. Using this approach requires tailoring skills, a significant time investment, and experience with physical simulation tuning. Sketch-based garment modelers [Decaudin et al. 2006; Turquin et al. 2007; Robson et al. 2011] generate a 3D garment model from user-sketched 2D silhouettes of the garment. This significantly speeds up and simplifies the design process. However, these methods do not achieve the same garment complexity and realism as the traditional work-flow, and still require a significant amount of user input per outfit. Thus they do not replace an automatic transfer method.

Interactive garment modeling systems [Volino et al. 2005; Umetani et al. 2011] allow for instant 3D feedback in response to changes in garment patterns by using a real-time physics-based simulation. Umentani et al. [2011] use such an interactive setup to quickly adapt patterns to a new character. However this process still relies on the user's ability to make the right design choices in order to achieve the desired look for the transferred garment. Similarly, the modeling framework of Harmon et al. [2011] helps resolve contacts and intersections in an interactive modeling environment but leaves the design choices up to the user.

Garment Transfer or Grading: The easiest way to transfer a garment from one character to another is to apply a physical simulation to place the garment on the new character. This solution is clearly inadequate, and produces a result that looks like a person wearing someone else's wrong size garment. In real-life, garment grading is applied to the $2 \mathrm{D}$ sewing patterns and follows a complex set of high-level rules that vary significantly for different garment styles [Moore et al. 2001]. In this process tailors first aim to scale the patterns to reflect changes in a number of key distances between the source and target wearers: hip or waist circumference, arm length, neck to waist length, etc. The challenge in this step is that distances along parallel curves can scale nonuniformly, requiring subtle, uneven, changes in pattern shape (see the skirt patterns in Figure 2). Consequently, this process remains
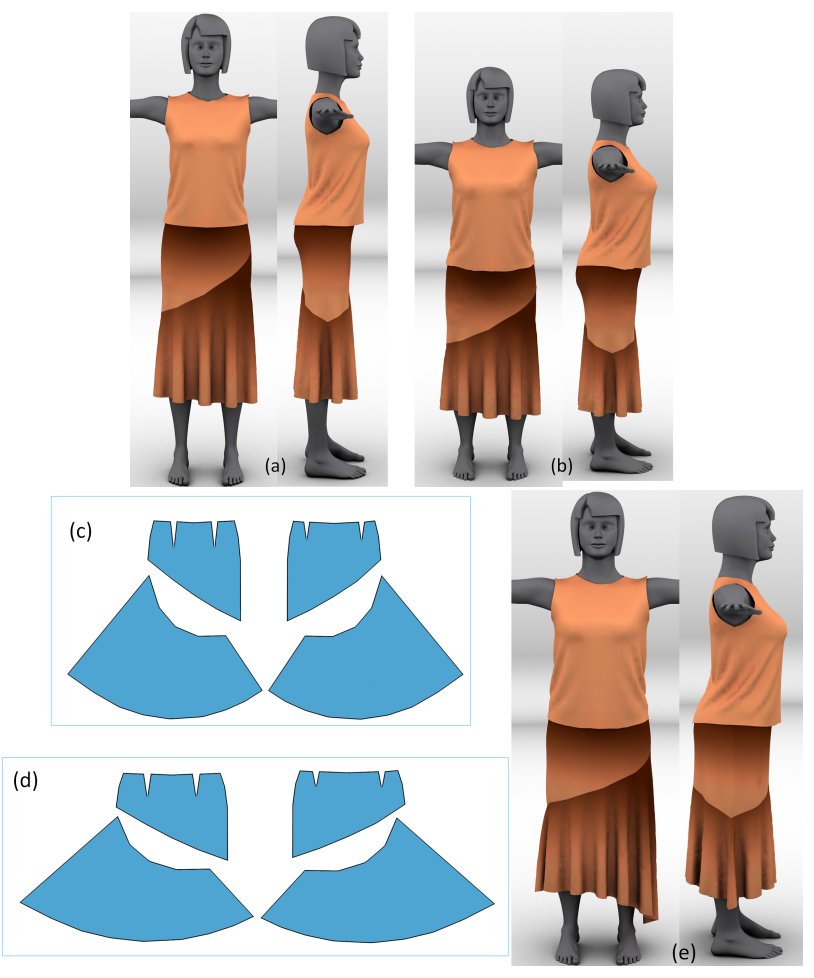

Figure 3: (top) Automatic transfer of a skirt inspired by Figure 2 from a tall model (a) to a short and stocky one (b). (bottom) Scaling the skirt patterns $(c)$ naively according to difference in height and hip circumference $(d)$ results in uneven hem length and baggy skirt (e). 


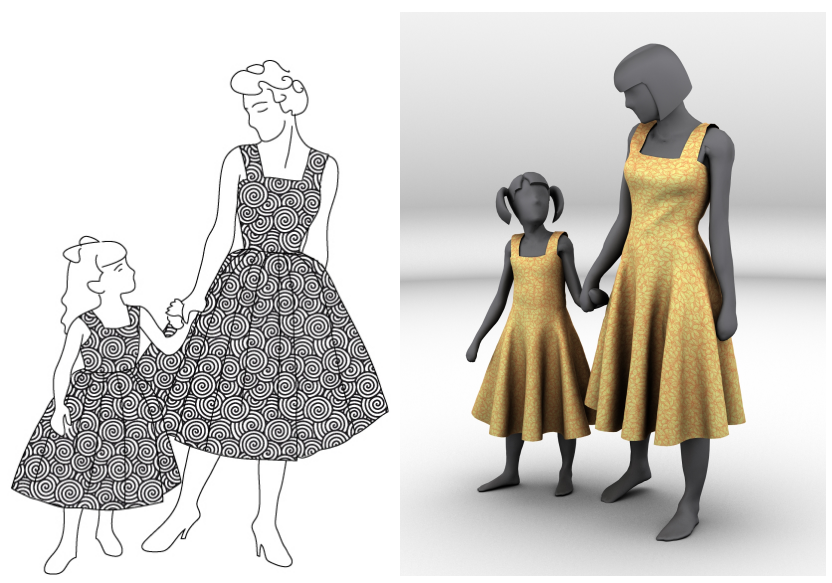

Figure 4: Mother-daughter dresses: (left) traditional grading; (right) our variation. (C)Laurence Boissieux 2012

manual and knowledge-intensive, as the designers need to decide how to preserve the original design while adjusting these distances. Computer-aided grading software, e.g. [eTelestia ; Optitex ; FashionCAD ], attempts to simplify this step by providing users with easy access to key-distance based sizing tables and automated grading rules. These systems are largely limited to a pre-defined set of grading rules and rely on user expertise to select the appropriate rules for each garment. Since a small set of distances cannot not fully describe the difference in proportions and body shape between people, a further refinement step is often necessary to obtain the desired look. This step involves a physical fitting process followed by additional alterations. For virtual characters who typically do not conform to standard body size tables, this process is particularly challenging as even the distance based scaling must typically be done completely manually.

As grading is a highly useful tool in generating both real and virtual garments, attempts have been made to automate it by operating directly in 3D. Several methods, e.g. [Wang et al. 2005; Wang et al. 2007], use variations of skinning techniques to do so. However, as pointed out by Meng et. al. [2012] and illustrated later on in Figure 13 skinning significantly changes the shape of loose garments, violating both the original design intent and plausibility. Consequently, Cordier et al [2003] apply skinning only to tight areas of the garment. They then globally scale the garment patterns for the loose parts along two major axes based on changes in wearer's height and hip circumference. Such axial scaling often results in poorly shaped garments (Figure 3, bottom) and is inconsistent with the much more complex process used by professional graders (Figure 2 ). By smoothly modifying the entire garment surface our method is able to preserve the source design (Figure 3, top). To preserve the design Meng et al. [2012] introduce an interactive transfer system where users correct a skinning generated garment by manually tracing the desired garment outlines in a number of views. Instead, we aim for a fully automatic design-preserving solution.

Resizing and Transfer (non garment): As part of the effort to simplify the creation of virtual 3D content, researchers have developed a variety of transfer and resizing techniques for different types of data. Properties to be transferred between different shapes range from textures and geometric details [Kraevoy and Sheffer 2004; Takayama et al. 2011] to deformation or animation [Sumner and Popovic 2004; Kircher and Garland 2006; Ben-Chen et al. 2009]. Our work follows this trend, extending the range of transferable content.
Deformation and geometry transfer methods, e.g. [Kircher and Garland 2006; Ben-Chen et al. 2009; Sumner and Popovic 2004; Bokeloh et al. 2011] often use a notion of shape preservation. However, the formulations they typically use penalize shear. These formulations cannot be used in a garment transfer setup where, as discussed in Section 3, proportional shearing based on changes in body proportions is not only unavoidable, but in fact often desirable. To realistically resize man-made objects, Kraevoy et al. [2008] minimize changes in surface normal. Their observation about normals reflecting the global shape directly applies to our setup (Section 3). However, our challenge is quite different due to the other considerations that garment transfer must satisfy. Specifically their volumetric solution approach cannot support the constraints arising from proportionality and plausibility which need to be balanced against shape preservation.

\section{The Geometry of Garment Grading}

Before developing a garment transfer method we must ask what a user's expectations from a transferred garment are [Moore et al. 2001]. A common statement of these transfer, or grading, requirements is that "the purpose of grading is to proportionally increase or decrease the size of a pattern, while maintaining shape, fit, balance, and scale of style details." [Threads ]. The following sections discuss the geometric formulation of these criteria, and then give an overview of our method.

\subsection{Grading criteria}

Proportion and Scale: The proportionality, or proportional increase/decrease of size, requires the resized garment to preserve the relative location of garment features with respect to the character's limbs and body. For instance: a knee-length skirt needs to remain knee-length independent of a change in a character's height, a dress waistline needs to remain on the waist, and a side zipper need to stay on the side. The proportional scaling of details reinforces proportionality, requiring not only to preserve feature location but to scale those appropriately. From a geometry processing viewpoint, proportionality and scale combined can be paraphrased as a single relative location requirement, where we associate a reference location on the body with each point on the garment and preserve the direction from one to the other during transfer, preventing the garment from sliding along or twisting around the body.

Shape: The shape preservation criterion requires grading to preserve the overall look or design of the input garment. E.g. a straight skirt should remain straight, boot-cut jeans should retain the bootcut, and so on. The mother-daughter dresses in Figure 4 (right) provide an illustration of shape preserving manual grading which successfully handles large differences in proportions. From our observations of professionally graded garments, confirmed in a discussion with a fashion designer, expert graders allow for significant changes in the geometry of the garments to accommodate changes in proportions but aim to preserve the slope or tangent plane orientations across the garment surface whenever possible. Thus for instance the hem width of bell-bottom jeans designed for a taller person is increased proportionally to height to maintain the bell slope. Consequently, we restate shape preservation in geometric terms as preservation of garment surface normals.

Fit: The fit criterion reflects preservation of local distance between the garment and the wearer. As discussed above, the distance to the body in loose areas is often expected to change significantly to preserve shape; hence, this criterion is largely enforced in tight regions. Even for these regions fit preservation is often a 

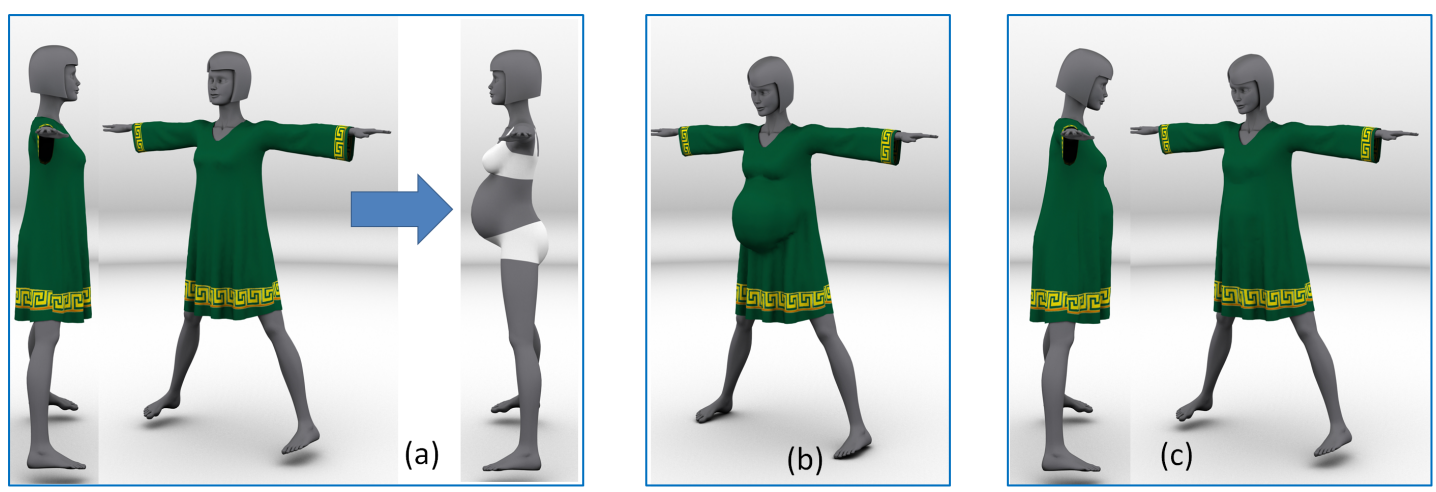

Figure 5: Algorithm Overview: Given the dressed source character and a target one (a), we first proportionally scale the $3 D$ garment to obtain a transferred version that satisfies proportionality and fit $(b)$. Then, an iterative optimization process combines the source garment normals and the positions generated by the scaling to obtain a design preserving transferred garment (c).

design choice, a designer may selectively relax fit in some locations to more closely adhere to the source garment shape. A typical example is transfer of a skirt or pants to a fuller person, where designers typically loosen the garment on the lower half of the belly to achieve a visually skinnier look [Freeth 2011]. Geometrically, fit preservation can be expressed as forcing the garment points that are very close to the source character's body to remain close to the target character after the transfer.

Balance: Lastly, the term balance refers to the hang of the garment around the body which is largely controlled by the orientation of the patterns with respect to the weave directions of the selected fabric. As such, it is orthogonal to the geometric considerations involved in transferring a 3D virtual garment, where one can always assume that the patterns remain correctly oriented.

In addition to the criteria above, we must consider two extra plausibility criteria that are implicit in real garment grading.

Manufacturability: Real garments need to be manufactured from planar fabric panels. As real-world fabric has a limited stretchability, the Gaussian curvature of the transferred 3D garment has to be bounded in the interior of each panel. The shape preservation metric previously discussed implicitly keeps the curvature low, since for a surface with a priori low Gaussian curvature, preserving normals implicitly preserves curvature. However, manufacturability needs to be explicitly considered when processing regions where fit is enforced. In these areas the garment follows the shape of the body and therefore can be affected by increase in body surface curvature, see Figure $8(a, b)$. To preserve manufacturability professional graders either selectively relax the fit thus reducing curvature, or introduce complex changes to patterns, such as multiple darts and gussets, to maintain the fit. In this paper we opt for the former option, selectively relaxing fit to minimize curvature increase.

Collision Avoidance: Real garments clearly cannot intersect the wearer's body or underlying clothing layers. Thus when transferring a garment to a larger person, designers increase pattern size to preserve shape and avoid fabric stretching or worse tear.

\subsection{Method Overview}

Our garment transfer method operates directly on 3D garments and finds a satisfactory balance between the criteria listed above: shape, relative location, fit, manufacturability, and collision avoidance.
The input to our method is a dressed source character model, possibly with the associated $2 \mathrm{D}$ clothing patterns, and a target character, all represented by manifold triangular meshes. We assume that the characters are posed in a similar pose, and that a dense correspondence or cross-parameterization is predefined between them. We also assume that both characters are rigged, i.e. have an animation skeleton. There are a large number of cross-parameterization methods, especially when it comes to rigged models, which can be easily used to satisfy these requirements [Sheffer et al. 2006; Chang et al. 2006].

We break the transfer process into two steps (Figure 5): a skinninglike initialization that generates a proportionally scaled reference garment (Section 4) and a global design preserving optimization (Section 5). To efficiently preserve the shape of the source garment, we restate normal preservation in terms of per-triangle transformation gradient. We introduce a transformation gradient based formulation which balances normal, relative location, and fit preservation and can be solved using an efficient iterative solver (Sections 5.1 and 5.2). In Sections 5.3 and 5.4, we show how to combine this minimization with collision avoidance and multi-layer garment processing, leading to the final iterative algorithm that generates the desired transferred garment.

\section{Proportional Scaling}

The first step of our method generates a proportionally scaled version of the input garment which satisfies the relative location and fit criteria (Figure 5, b). This scaled version is then used as a starting point for the normal preserving optimization which generates the target transferred garment. Our proportional scaling method can be seen as an extension of smooth skinning, where the combination of constant positions in several skeleton frames is replaced by a combination of constant offset vectors from a set of adequately chosen reference points on the character model. Using skin-based offsets allows our method to cope with changes in body shape, such as arm thickness, in addition to handling changes in skeletal proportions.

\subsection{Selecting reference points}

To perform the proportional scaling we first associate each garment vertex $p_{g}$ with pairs $\left(p_{b}, p_{m}\right)$ of relevant bone and character skin points. We call these pairs reference points. Note that using the closest skin point as a reference [Cordier et al. 2003; Meng et al. 2012] is not necessarily the best choice (see Figure 6, left), as it can cause points to slide along the body on the target character. As- 
signing references orthogonally to the local bone directions reduces such sliding, resulting in better relative location preservation, most notable along garment boundaries. Therefore, the reference point pairs we select correspond to local minima of a function expressing distance to the character model in a direction orthogonal to the local bone (see Figure 6), computed as follows.

Given a garment vertex $p_{g}$, we first compute its closest points $p_{b}$ on each bone of the animation skeleton ( $p_{b}$ is possibly an extremity). For each bone, we define $p_{m}$ as the input character point closest to $p_{g}$ along the segment $\left[p_{b}, p_{g}\right]$. Such a point necessarily exists since $p_{b}$ is inside, and $p_{g}$ outside the character model. To select one or more reference points we consider both the distance from $p_{g}$ to each of the intersections $p_{m}$ and the angle between the bone axis $v_{b}$ and the vector $p_{g}-p_{b}$ as we prefer these two vectors to be orthogonal. The pairs $\left(p_{b}, p_{m}\right)$ we select as reference for $p_{g}$ are those that minimize a combination of these two metrics $\left\|p_{m}, p_{g}\right\| e^{-\frac{\left\langle v_{b}, v\right\rangle^{2}}{\sigma^{2}}}$, where we set $\sigma=0.1$. In most cases this function has a clear single minimum (Figure 6, right), which we select as the reference. When two identical or very close minima exist, we assign both as references. This is typically the case in areas in-between the legs of the character or under the arms (see Figure 6, center). Finally, each point $p_{g}$ is associated with an offset $o=\left\|p_{g}-p_{m}\right\|$ for each reference pair $\left(p_{b}, p_{m}\right)$.

\subsection{Proportional scaling using offset vectors}

We use the computed reference points and offsets to obtain the proportionally scaled locations $\hat{p_{g}}$ for each garment point with respect to the target character. First, for each reference pair $\left(p_{b}, p_{m}\right)$ on the source character, we use the cross-parameterization to obtain the corresponding pair of points $\left(\hat{p}_{b}, \hat{p}_{m}\right)$ on the target. Each point $p_{g}$ has one, or more, reference pairs. If it has a single reference pair $\left(p_{b}, p_{m}\right)$, we set $\hat{p_{g}}=\hat{p_{m}}+o * v_{b m}$, where $v_{b m}$ is a unit vector in the direction of $\left(\hat{p}_{b}, \hat{p}_{m}\right)$. If there are several reference pairs for the garment vertex $p_{g}$, we use a weighted sum of the positions dictated by each reference, using non-linear weights to get a sharp transition, $w_{1}=\frac{\arctan \left(5 *(t-0.5)+\frac{\pi}{2}\right)}{\pi}$ and $w_{2}=1-w_{1}$, where $t$ is the coordinate of the projection of the garment vertex $p_{g}$ on the segment between the two relevant bone reference points.

Figure 5, (b) shows the output of the proportional scaling step. Note that garment borders and other features keep their relative location with respect to the character's body and limbs, while the use of skin based offset accounts for changes in body shape between the input and output character, preserving fit. At the same time, the results clearly do not account for shape preservation, necessitating

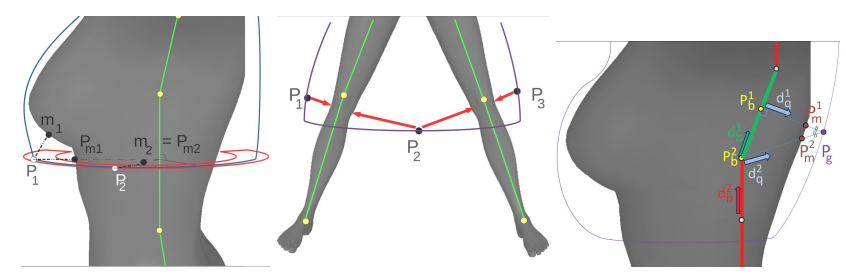

Figure 6: (left) Selecting closest skin points $m_{1}$ and $m_{2}$ as reference points for $p_{1}$ and $p_{2}$ is suboptimal, as they can move apart on the target character, causing an undesirable vertical displacement. Selecting the orthogonal direction, will map both points to a circle around the same bone point, preserving the relative height. (center) Association to multiple reference points is necessary to correctly account for regions roughly equidistant to multiple bones. (right) Association must depend on angle as well as distance to nearest intersection, giving preference to $p_{m}^{1}$ over $p_{m}^{2}$.

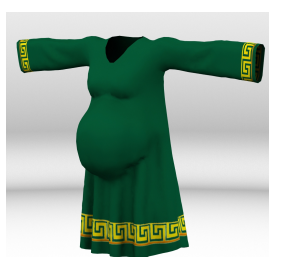

(a)

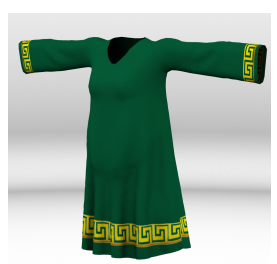

(b)

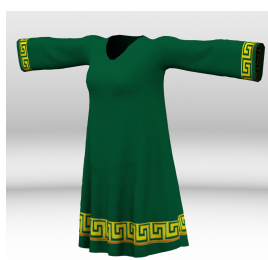

(c)
Figure 7: Intermediate stages of shape preserving transfer for the tunic in Figure 5: (a) initial proportional scaling output forms an offset surface around the belly; (b) after one iteration of shape preserving transfer; (c) after convergence (6 iterations) with no collision testing $(c)$. With collision avoidance added-in the final result in Figure $5(c)$ is obtained.

the main step of the algorithm described next.

\section{Shape Preserving Garment Transfer}

\subsection{As-2D-as-possible Deformation}

Preservation of garment shape requires the normals of the garment mesh triangles to remain constant or near-constant during transfer. In general, expressing normal preservation as a function of vertex positions leads to a non-linear, hard to control optimization. Hence, existing deformation and resizing methods, e.g. [Bokeloh et al. 2011] tend to penalize both out-of-plane rotations and $2 \mathrm{D}$ deformations. To penalize all rotations, [Kraevoy et al. 2008] use a volumetric formulation with global scaling constraints. Their approach can not be extended to our setup as we require a much finer level of control. To efficiently penalize rotations, while allowing for $2 \mathrm{D}$ deformations we express normal preservation via transformation gradients. This formulation allows for a solution mechanism based on iterative linear minimization of a quadratic functional. Contrary to previous gradient based methods, which aim to either preserve known transformation gradients [Sumner and Popovic 2004] or to search for as-rigid-as-possible ones [Igarashi et al. 2005; Sorkine and Alexa 2007], we search for as-2D-as-possible transformation gradients.

Following Sumner et al. [2004] we label the vertices of each source garment triangle $t$ as $p_{1}, p_{2}$ and $p_{3}$ and add a virtual vertex $p_{4}$ computed by offsetting $p_{1}$ by the triangle normal. We then define the $3 \times 3$ matrix representing the local triangle frame as $P^{t}=\left(p_{4}-p_{1}, p_{4}-p_{2}, p_{4}-p_{3}\right)$. The gradient of the triangle transformation from the source to the target garment can then be written as $\tilde{P}^{t}\left(P^{t}\right)^{-1}$ where $\tilde{P}_{i}^{t}$ is the local frame after the deformation. Normal preservation for all the mesh triangles is thus expressed as the minimization of:

$$
E_{\text {shape }}=\sum_{t}\left\|\tilde{P}^{t}\left(P^{t}\right)^{-1}-T^{t}\right\|_{F}^{2}
$$

where $\|.\|_{F}$ is the Frobenius norm for matrices, and the unknown target triangle gradients $T^{t}$ are constrained to be $2 \mathrm{D}$-only transformations defined in the plane of the source triangle, i.e. applying $T^{t}$ to $t$ preserves the triangle's normal.

Solver: The numerical challenge we face is to enforce the 2Donly constraints on $T^{t}$ while solving for both $\tilde{p}$, the target vertex positions, and $T^{t}$, the target gradients, subject to the additional constraints listed in Section 5.2. To compute a solution we use an iterated least-squares process which alternates between updating 
$T^{t}$ while keeping $\tilde{p}$ fixed, and vice versa. We start by setting $\tilde{p}$ to the positions $\hat{p}$ of the vertices after the proportional scaling based initialization (Section 4). We then iterate the two subsequent steps.

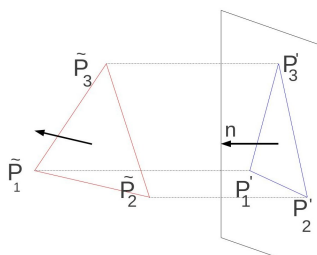

Updating $T^{t}$ : To update $T^{t}$ given the current value of $\tilde{p}$, one option is to perform $\mathrm{QR}$ decomposition of the gradient $\tilde{P}^{t}\left(P^{t}\right)^{-1}$ and use the nonrotational component as the new 2Donly transformation gradients. However such decomposition discards the in-plane rotations, which in our case we want to allow. Instead we compute $T^{t}$ as follows (see inset figure): we project each triangle to the corresponding plane using the reference normal $n^{t}$ on the source garment:

$$
p_{i}^{\prime t}=\tilde{p}_{i}^{t}-<\tilde{p}_{i}^{t}, n^{t}>n^{t}
$$

obtaining a local frame $P^{\prime t}=\left(p_{4}^{\prime}-p_{1}^{\prime}, p_{4}^{\prime}-p_{2}^{\prime}, p_{4}^{\prime}-p_{3}^{\prime}\right)$. Typically, $\left\|\tilde{P}^{t}\left(P^{t}\right)^{-1}-P^{\prime t}\left(P^{t}\right)^{-1}\right\|<\left\|\tilde{P}^{t}\left(P^{t}\right)^{-1}-T^{t}\right\|$. If this is the case we set $T^{t}=P^{\prime t}\left(P^{t}\right)^{-1}$, otherwise $T^{t}$ is unchanged. Consequently the update step is guaranteed to never increase the value of the optimized functional. Since the same is true for the vertex solve, the optimization is guaranteed to converge.

Vertex solve: With $T^{t}$ fixed, the solution for $\tilde{p}$ minimizing the quadratic equation (1) reduces to a simple linear system, which we solve in combination with the constraints detailed below. Since the positions do not change significantly between iterations, we use an iterative CG solver with the previous positions as an initial guess.

\subsection{Accounting for relative location and fit constraints}

To generate a properly transferred garment the shape preservation term described by Equation 1 needs to be combined with terms enforcing preservation of relative locations and fit. Both of these are by construction satisfied by the vertex positions $\hat{p}$ of the proportionally scaled garment computed by the initialization step (Section $4)$.

To introduce these considerations into the shape-preserving formulation we consider the local frame(s) defined by the relationship between each vertex $\hat{p}$ on the proportionally scaled garment and its associated reference skeleton point(s) $p_{b}$. Each frame is formed by the directions $d_{b}$ of the corresponding skeleton bone, the unit vector $d_{q}$ collinear to $\left(p_{b}, \hat{p}\right)$, and their cross product $d_{t}$ (by construction $d_{q}$ is typically orthogonal to $d_{b}$ ).

To enforce relative location preservation we use the following term

$$
E_{\mathbf{r l}}=\sum_{\tilde{p}} \alpha_{p}\left(<\tilde{p}-\hat{p}, d_{b}>^{2}+<\tilde{p}-\hat{p}, d_{t}>^{2}\right) .
$$

The two components of the sum aim at preventing the garment from sliding along or twisting around the skeleton respectively. For interior vertices in loose regions we use a low $\alpha_{p}=0.5$ prioritizing shape preservation at the expense of minor inaccuracy in relative locations. Along garment boundaries and seams where any twisting or displacement would be very noticeable, as well as in the tight regions, we set $\alpha_{p}=1000$. As relative location does not dictate any constraints on the distance between the character's body and the garment, the component of $\tilde{p}-\hat{p}$ aligned with $d_{q}$ is unconstrained.

To enforce fit in tight regions, specified as described below, the relative location term is augmented with a fitting term, explicitly constraining this distance,

$$
E_{\mathrm{fit}}=\beta \sum_{t \in F} \sum_{\tilde{p} \in t}<\left(\tilde{p}-\hat{p}, d_{q}>^{2}\right.
$$

where the set $F$ contains the triangles in the regions deemed tight, and $\beta=1000$. The triangles in $F$ are also removed from the shape energy term $E_{\text {shape }}$ as in this case there is no reason to preserve their normals.

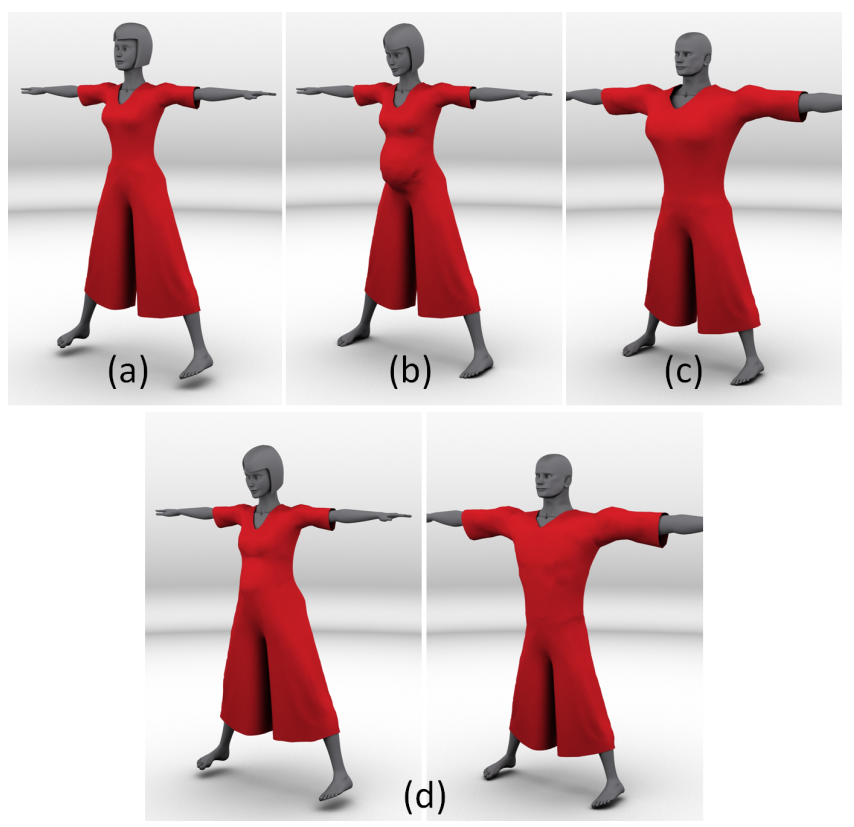

Figure 8: The impact of fit settings: (a) source; (b) enforcing fit across all the tight regions on a pregnant female leads to undesirable increase in curvature and focuses attention on the belly; $(c)$ with no fitting enforced the garment transferred to a male looks implausible. (d) Our results for both models with fit enforced selectively.

Using the two terms above, the energy functional to be minimized in the iterative process described in Section 5.1 is set to:

$$
E=E_{\text {shape }}+E_{\mathbf{r l}}+E_{\text {fit }}
$$

Given the degree of freedom provided by varying the distance to the character's body, we typically can simultaneously preserve both relative location and shape in the loose regions pretty well.

Selecting the Tight Regions: As stressed in Section 3, in reallife grading the selection of the region where fit should be preserved is a design choice [Freeth 2011]. The simplest selection would be based on a distance threshold. However this choice is problematic when the body curvature grows significantly between the source and target characters - e.g. from a regular female to a pregnant one (Figure 8, b). In this case, preserving tightness can increase the garment's Gaussian curvature making manufacturing a challenge and creating an unappealing look. Instead, we opt for the selective relaxation technique described in Section 3, relaxing fit in areas where the normal to the source garment points downward or sideways. We thus assign to $F$ triangles with all vertices located closer than a distance threshold from the model, and whose normals $n^{t}$ on the source garment are pointing upward. This strategy leads to consistently believable transferred garments, such as the overalls in Figure 8 (d). Note, that completely relaxing the fit across 
the model can lead to implausible results (Figure 8, c), with the male character looking like he wears a bra. The reason is that lacking physical context the method cannot distinguish between upward pointing garment areas which are a product of garment-body interaction, as is the case for the overall, and those formed by design (e.g. through bra type seaming). Our tightness setup assumes the former scenario, as it is a more likely one.

\subsection{Handling collisions}

The transfer process as described so far does not explicitly prevent garment interpenetrations with the target character or selfintersections. Self-intersections are very rare and and can be easily resolved by any standard post process that moves vertices apart without any changes to the overall garment design. In contrast, body-collisions typically occur when transferring a garment to targets with more protruding parts than the source (Figure 9, a) and indicate a need to add more fabric to accommodate the change in body shape. Note that collisions can only occur in the loose regions of the garment, as the fit constraint prevents interpenetrations in tight regions. To add more fabric and prevent collisions during transfer we take advantage of the local frames $\left(d_{b}, d_{q}, d_{t}\right)$ associated with each vertex (Section 5.2). Using these frames collision avoidance can be paraphrased as

$$
<\tilde{p}-p_{m}, d_{q}>\geq \epsilon
$$

In other words, the garment has to be farther away from the bone than the skin of the model. Combining this constraint with strong preservation of relative location, one can theoretically avoid all in-

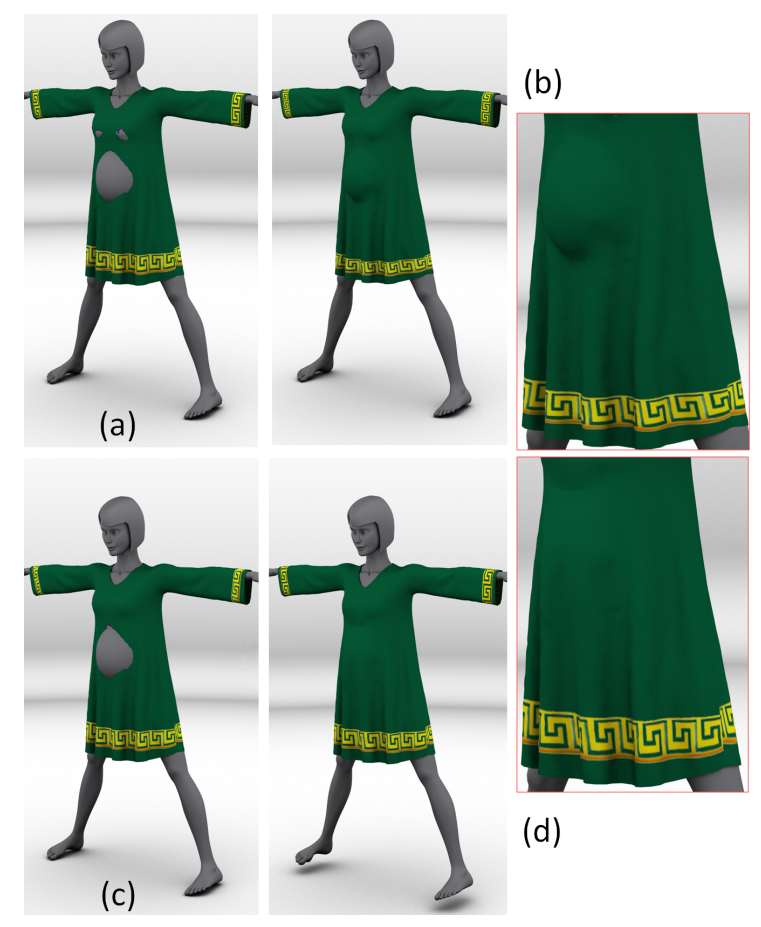

Figure 9: Collision resolution order: (a) unconstrained solution with step one collisions highlighted, (b) converting all collisions into equality constraints at once leads to undesirable artifacts on the belly (see inset zoom). (c) Incremental solution within one iteration resolves all collisions on the chest (step two collisions highlighted) and in a few iterations generates better transfer results with all collisions resolved $(d)$. tersections with the body. The practical challenge is in enforcing such inequalities efficiently.

Solution Mechanism: To implement efficient collision avoidance we opt for an active set approach especially tailored for our needs. Standard active set methods [Nocedal and Wright 2006] replace inequalities by strategically selected equality constraints, using an iterative addition and removal process. In our case,we leverage knowledge about the problem setup at hand, to develop an efficient solution method where only strictly necessary equalities are added and consequently never removed. This modification makes the solution both faster and robust.

We first optimize the energy functional in Equation 4 with no collision constraints, and then iteratively introduce collision-resolving equality constraints and repeat the optimization as long as collisions persist. Specifically, we observe that in our setup resolving collisions higher-up along the body often resolve those below, while the opposite practically never happens. In general, once we move vertices away from the body, shape preservation pulls those below away as well, while vertices higher up are held in place due to fit constraints typically enforced in the tight regions on the shoulders. This is likely due to garment interaction under gravity. Knowing this, we use a top to bottom equality enforcement strategy. We obtain best results by adding constraints very gradually, two by two (to account for symmetries often present in garment models). After each collision processing step, we repeat the optimization (Equation 4). This iterative process leads to better results than introducing the constraints all at once, see Figure 9 (b) versus Figure 5 (c).

The actual collision detection can be done very efficiently by intersecting the segment(s) $\tilde{p}-p_{b}$ (where $p_{b}$ is the reference bone point associated with $p$ ) with the mesh representing the target character and performing an inside/outside test by comparing skeletongarment vs skeleton-character distances. While in theory the garment can intersect other areas on the skin, this had never happened in practice. In the case of penetration, we recompute $\hat{p}$ by projecting the vertex a small distance outside the model along the current segment $\tilde{p}-p_{b}$ and add the highest triangle adjacent to the vertex to $F$ (Figure 9, c), treating the triangle as tight fitting for further computations.

Even with the very gradual introduction of equalities, the collisions are typically resolved in just a few iterations; see Table 1 for permodel counts.

\subsection{Extension to layers of garments}

Real-life outfits often consist of multiple layers either fully separate, such as a dress with an under-skirt (Figure 1), or stitched together, such as a dress with multiple ruffle layers (Figure 10), or a jacket with outside pockets . Our transfer method easily extends to this case. We incrementally process each fabric layer, starting with the layer closest to the body and proceeding to the outer one. At each stage, we treat the already processes layers as part of the current character model. The outermost surface of this model is then used to compute the proportionally scaled version of the next source layer, used to initialize the subsequent optimization process.

\subsection{Pattern Extraction}

To obtain patterns for the transferred garment, we cut it along the seams indicated on the source and use the $\mathrm{ABF}++$ parameterization method [Sheffer et al. 2005] to parameterize the resulting charts. We found that this approach provides an optimal time-quality tradeof. It performs equally well to more time consuming stretch minimization techniques. At the same time it creates better, more sym- 


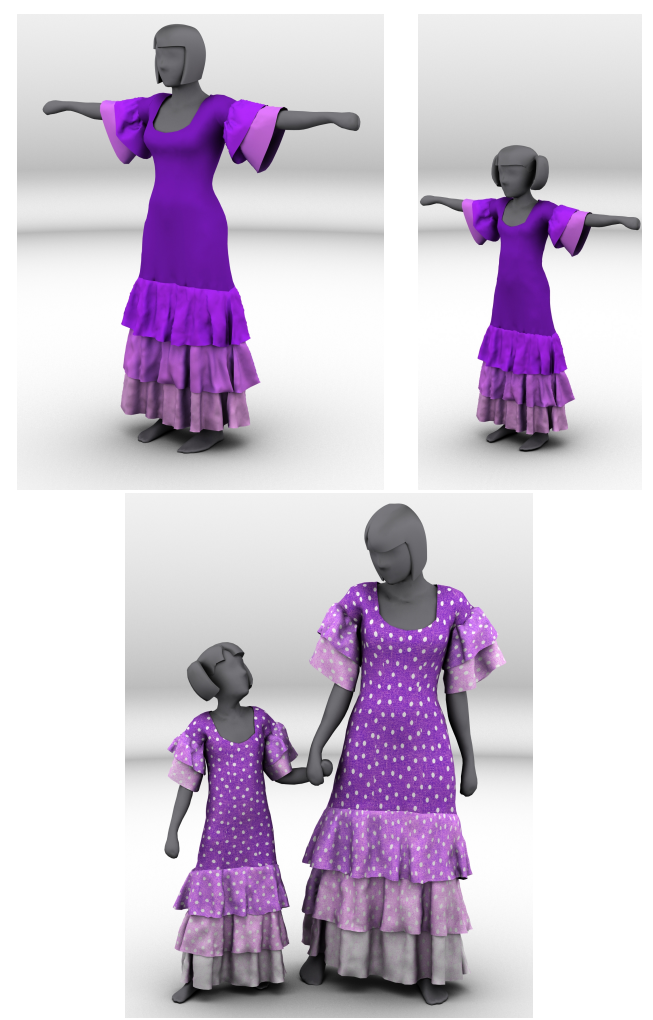

Figure 10: A multi-layer flamenco dress transferred from a woman to a girl. The undistorted texture highlights our method's ability to automatically generate appropriate patterns. (c)Laurence Boissieux 2012

metric, patterns than faster, linear methods [Sheffer et al. 2006] in cases where the source and consequently target garments are not perfectly developable. We automatically discard darts that are no longer necessary, i.e. ones effectively closed by the parameterization. The $L^{2}$-stretch [Sheffer et al. 2006] between the garments and the patterns we computed was in the range of 1.0002 to 1.003 (ideal value is 1), indicating negligible distortion. Figures 1 and 11 show patterns created using our approach. Patterns created this way are well suited for texturing or physical simulation. However they tend to have somewhat wavy outlines, sub-optimal for actual manufacturing. To regularize those, one can apply standard methods which approximate nearly straight outline segments by straight lines, and nearly circular ones by perfect arcs [Mehra et al. 2009].

\section{Results and discussion}

Throughout the paper we demonstrate the results of our method on a variety of models. We show results both in the canonical pose in which the transfer was processed (e.g. Figures 5, 12) and after simulation in alternative poses (e.g. Figure 1 and associated video). The latter demonstrate that, consistent with user expectations, the transfer process preserves design similarity between the source and target garments in general and not just in a particular pose. Figures 1 (left) and 10 show believable transfer of complex layered outfits between models with significant differences in proportions and body shape. Figure 1 (right) further reinforces the method's robustness to large changes in proportions and shape and highlights the suitability of our method for transferring garments to large crowds of highly diverse, often unrealistic, virtual characters. Figure 12 shows transfer of typical real-life garments to increasingly differ-

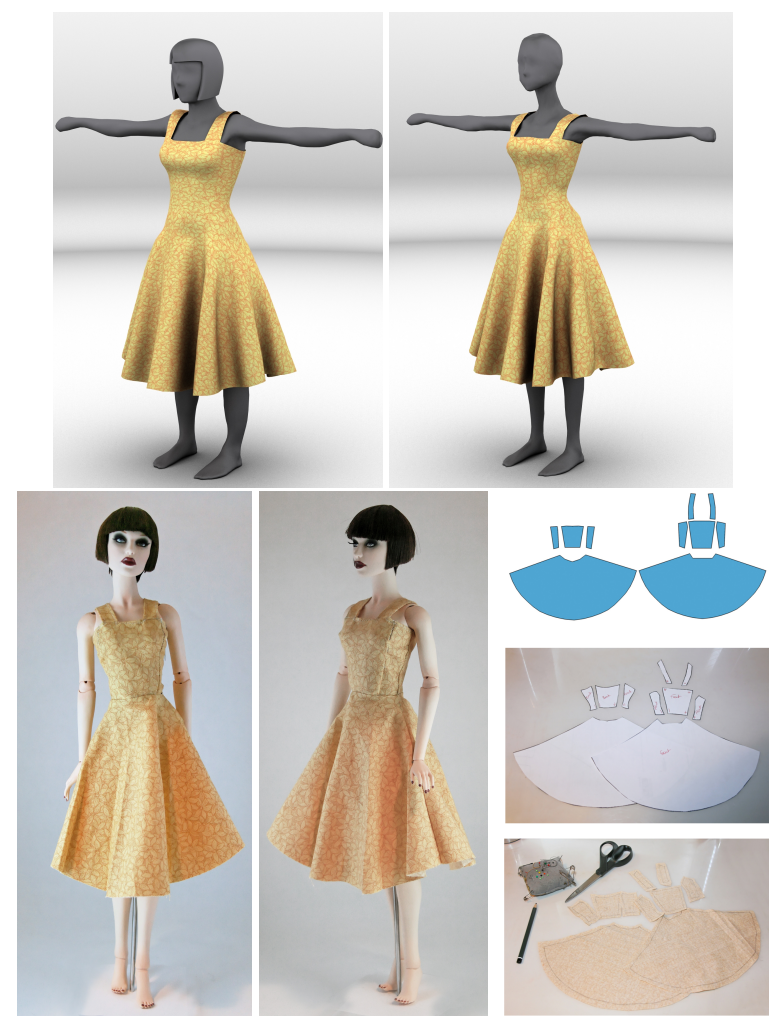

Figure 11: Validation on a real dress: we transferred the woman's dress on the top-left to a Numina doll model (c)Dollcis (top-right) and used the patterns to make an outfit for the real doll (bottom). The virtual source and real graded patterns for the front and back of the dress at bottom right. (c)Laurence Boissieux 2012

ent characters, creating results consistent with viewer expectation. The texture on the transferred models in Figures 4, 10 and others highlights preservation of manufacturability during transfer.

Validation: In addition to visual inspection by non-experts and amateur tailors, we had the results evaluated by a fashion designer, Nurit Perla (www.talia-designer-clothes.com), who concluded that our outputs satisfy all the criteria used in traditional grading. We further validated the approach by making a dress for an actual doll (Figure 11). We transferred the source dress to a 3D model of the doll, which has very different proportions from the source character, and then used the resulting patterns to make the actual dress. The resulting dress maintains the design and style of the source while nicely fitting the doll's dimensions, showcasing our method's applicability for transferring real-life garments.

Comparison: Existing garment transfer methods can be split into three groups: skinning based [Wang et al. 2005; Wang et al. 2007], axis-aligned scaling [Cordier et al. 2003], and user-assisted [Meng et al. 2012]. Figure 3 provides a comparison between axis-aligned scaling combined with physical simulation and our result. While the scaled skirt is baggy and has an uneven hem length, ours faithfully reflects the source design. Figure 13 provides a comparison of our result to those of [Wang et al. 2005] and [Meng et al. 2012]. The models were graciously provided by the authors. As clearly demonstrated by Figure 13 (b) skinning-like approaches create undesirable artifacts, avoided by our method. For similarly proportioned source and target models such as the dress in Figure 13 (top), 


\begin{tabular}{l|c|c|c|c|c} 
Model & $\# \Delta$ & runtime & init & \# iter. & $\#$ coll. \\
\hline Short woman dress & 8178 & $61 \mathrm{~s}$ & $15 \mathrm{~s}$ & 19 & 15 \\
Pregnant w. dress & & $189 \mathrm{~s}$ & & 64 & 57 \\
Man dress & & $99 \mathrm{~s}$ & & 34 & 18 \\
Pregnant woman Tunic & 9102 & $70 \mathrm{~s}$ & $16 \mathrm{~s}$ & 23 & 13 \\
Man over-all & 11631 & $181 \mathrm{~s}$ & $19 \mathrm{~s}$ & 46 & 34 \\
Pregnant w. over-all & & $112 \mathrm{~s}$ & & 28 & 18 \\
Short w. t-shirt/trousers & $6282 / 7563$ & $14 \mathrm{~s} / 36 \mathrm{~s}$ & $10 \mathrm{~s} / 10 \mathrm{~s}$ & $3 / 12$ & $0 / 9$ \\
Pregnant w. t-shirt/trousers & & $76 \mathrm{~s} / 137 \mathrm{~s}$ & & $39 / 57$ & $34 / 38$ \\
Man t-shirt/trousers & & $32 \mathrm{~s} / 32 \mathrm{~s}$ & & $10 / 10$ & $7 / 9$ \\
Girl 60s dress & 11864 & $86 \mathrm{~s}$ & $8 \mathrm{~s}$ & 20 & 13 \\
Doll 60s dress & & $171 \mathrm{~s}$ & & 43 & 38 \\
Girl layers & $6274 / 5979$ & $11 \mathrm{~s} / 10 \mathrm{~s}$ & $9 \mathrm{~s} / 9 \mathrm{~s}$ & $5 / 6$ & $0 / 2$ \\
& 5322 & $16 \mathrm{~s}$ & $9 \mathrm{~s}$ & 12 & 4 \\
Girl flamenco & 18560 & $76 \mathrm{~s}$ & $11 \mathrm{~s}$ & 12 & 5 \\
Girl dancer top/bottom & $4879 / 8809$ & $89 \mathrm{~s} / 17 \mathrm{~s}$ & $5 \mathrm{~s} / 3 \mathrm{~s}$ & $38 / 10$ & $32 / 6$ \\
Male dancer top/bottom & & $40 \mathrm{~s} / 23 \mathrm{~s}$ & & $14 / 15$ & $8 / 8$ \\
Tall dancer top/bottom & & $37 \mathrm{~s} / 21 \mathrm{~s}$ & & $13 / 13$ & $7 / 7$ \\
Fat dancer top/bottom & & $199 \mathrm{~s} / 49 \mathrm{~s}$ & & $81 / 36$ & $67 / 23$
\end{tabular}

Table 1: Result statistics (left to right): model, size in triangles (shown once per source input), transfer time, reference point computation (Section 4.1, done once per source model), number of iterations overall, and number of resolved collisions. For the flamenco dress the numbers for the three layers are combined.

the output garments of Meng et al. and ours are of comparable quality. Note however that Meng et al. use manually traced profile curves, with a subset of those shown in the figure, to generate the output dress. Our transferred dress is generated automatically. However, close examination reveals artifacts on the dress created by the user-assisted technique, not present in our outputs. The differences are drastically more pronounced when a dress is transferred form a non-pregnant to pregnant model (bottom). The result of Meng et al. retains the "skinning" effect around the belly, leading to an unnaturally looking and physically implausible result. In contrast, as demonstrated throughput the paper on this and other inputs our method can handle extreme changes in body shape and proportions, while preserving source design.

Statistics: Table 1 summarizes the statistics for our models. For typical virtual garments with five to twelve thousand triangles, our transfer process takes one to two minutes to generate the target outfit on a standard desktop PC (Intel Core 2 Duo T6400 2GHz). The runtime largely depends on the number of collisions that need resolution.

Limitations: The main limitation of our method is its sensitivity to the setting of the tight region tolerance, as unfortunately different input virtual models have different minimal offsets between the garment and the character, depending on the original modeling setup. Accordingly, the tightness tolerance needs to be adjusted depending on the model source. The output quality is also dependent on the quality of the cross-parameterization between the source and target characters. Lastly, our method is limited to manifold meshes; we did not find this to be a restriction, as most virtual garments are represented as such.

\section{Conclusion}

By automating garment transfer, our work greatly simplifies the generation of dressed characters, currently one of the more tedious and difficult task for computer artists. It enables transfer of both virtual and real-life garments between characters with very different body shape and proportions automatically adapting source patterns to fit the transferred garment. Our technique lets non-expert users to adapt pre-existing wardrobes to their characters of choice, removing the tailoring knowledge barrier. It is directly applicable to clothing crowds of arbitrary characters, as it requires no per-output tweaks. The two key components of our approach are a geometric formulation, and subsequent efficient optimization, of the set of criteria used in manual pattern-grading.

Our method has three main limitations. We rely on purely geometric considerations in the setting of the tight regions. Reverse engineering designer intent can be an interesting topic for future research. Similarly we ignore the impact of fine cloth mechanics on the shape of real-life or simulated output garments. However as demonstrated by the figures and the accompanying video our outputs retain the source design following simulation. Lastly, the method is sensitive to the quality of the cross-parameterization between the source and target models.

Possible improvements of the method include further speedup through the use of tailored solvers, such as [Ascher and Boxerman 2003] and pattern regularization consistent with tailoring approaches. It would be very interesting to apply our method to resizing of other 3D shapes, comparing the results with state of the art [Kraevoy et al. 2008; Bokeloh et al. 2011].

\section{Acknowledgements}

We would like to thank Nurit Perla for her valuable input and result evaluation and Paul Pham for permission to use the Numina doll. This research was partially funded by NSERC, Killam fund, MITACS and GRAND NCEs, and by the ERC advanced grant EXPRESSIVE.

\section{References}

ASCher, U., AND Boxerman, E. 2003. On the modified conjugate gradient method in cloth simulation. The Visual Computer 19, 7-8, 523-531.

Ben-Chen, M., Weber, O., And Gotsman, C. 2009. Spatial deformation transfer. Eurographics/ ACM SIGGRAPH Symposium on Computer Animation.

BoKeloh, M., Wand, M., Koltun, V., And Seidel, H.-P. 2011. Pattern-aware deformation using sliding dockers. ACM Transactions on Graphics (SIGGRAPH Asia 2011).

Chang, Y.-T., Chen, B.-Y., Luo, W.-C., And Huang, J.-B. 2006. Skeleton-driven animation transfer based on consistent volume parameterization. In Proceedings of Computer Graphics International 2006, 78-89.

Cordier, F., Seo, H., and Magnenat-Thalmann, N. 2003. Made-to-measure technologies for an online clothing store. IEEE Computer Graphics and Applications 23, 38-48.

Decaudin, P., Julius, D., Wither, J., Boissieux, L., ShefFER, A., AND CANI, M. 2006. Virtual garments: A fully geometric approach for clothing design. EUROGRAPHICS $2006 /$ E. Grller and L. Szirmay-Kalos 25.

ETELESTIA. www.etelestia.com/en/cad - pattern making - software.aspx.

FASHIONCAD. //www.fashioncad.net/.

FreEth, M. 2011. Maintaining design aesthetics : case studies investigating grading for body shape variation: the translation of garment designs to fit fuller figured women. Massey University, Wellington, New Zealand. 


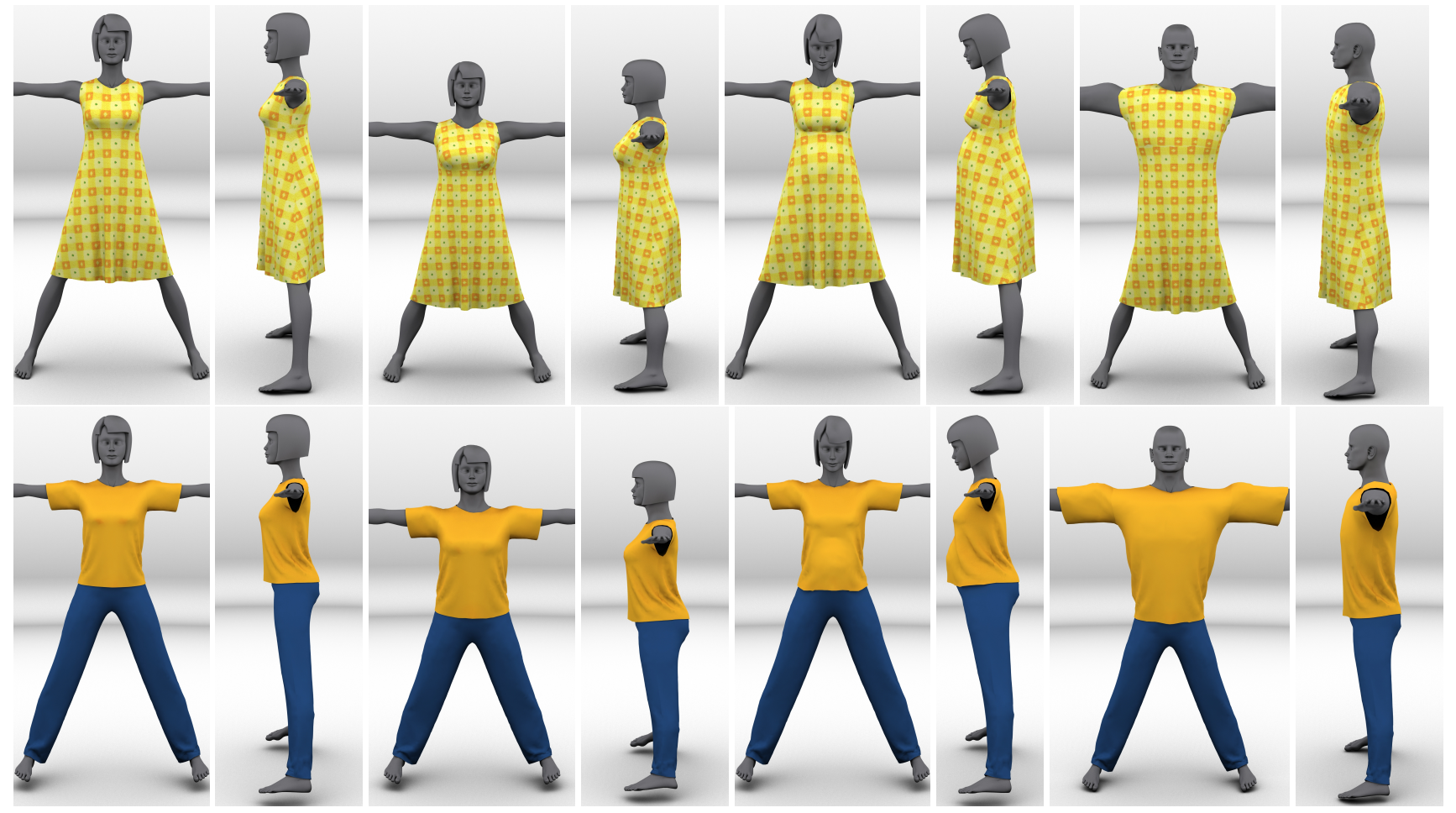

Figure 12: Transfer of garments to progressively more different models (front and side views): a stockier shorter female, a pregnant female, and an exaggerated male character. The texture on the transferred dresses was generated using the automatically computed patterns. Discontinuities (e.g. the line under the chest) reflect seam locations.
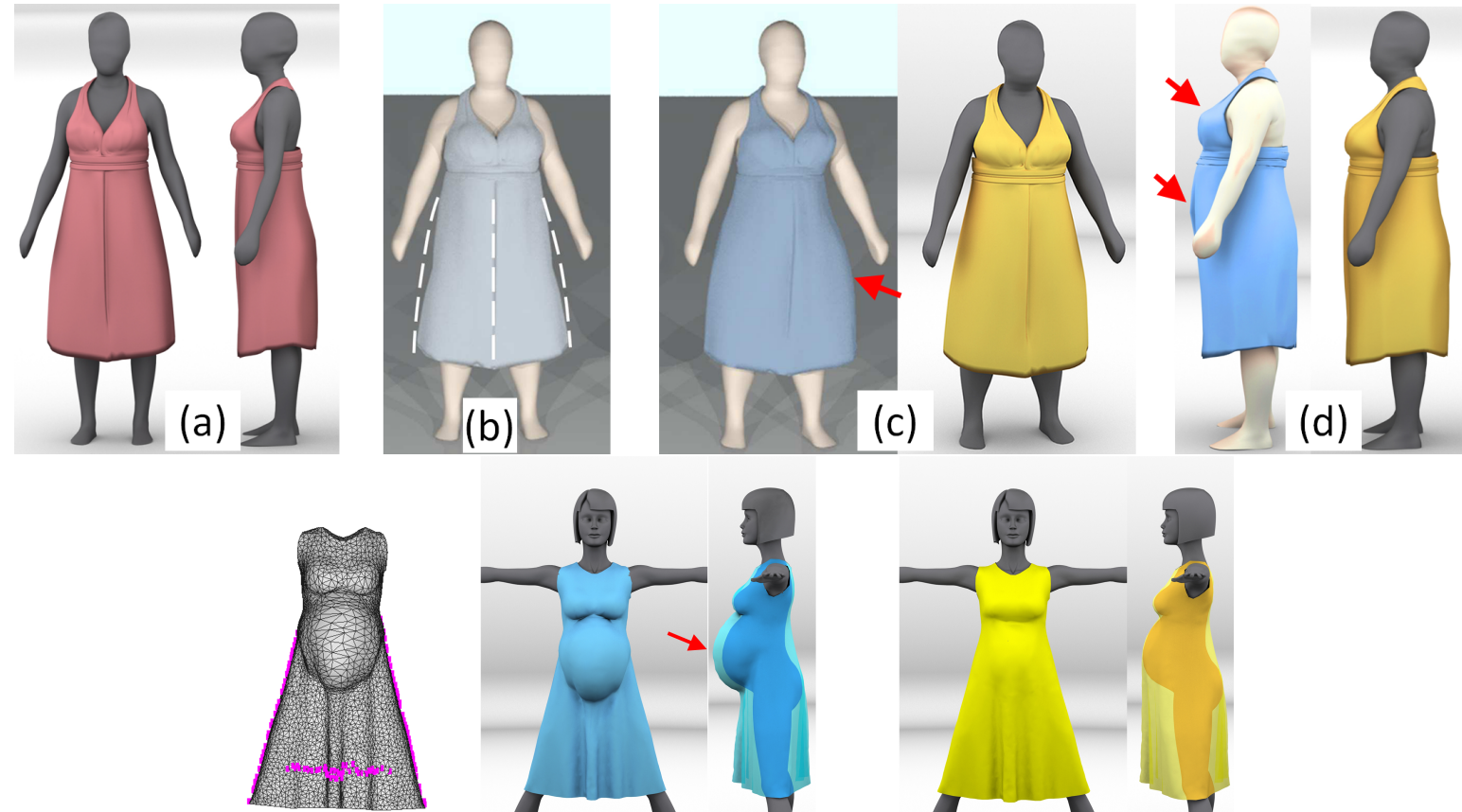

(e)
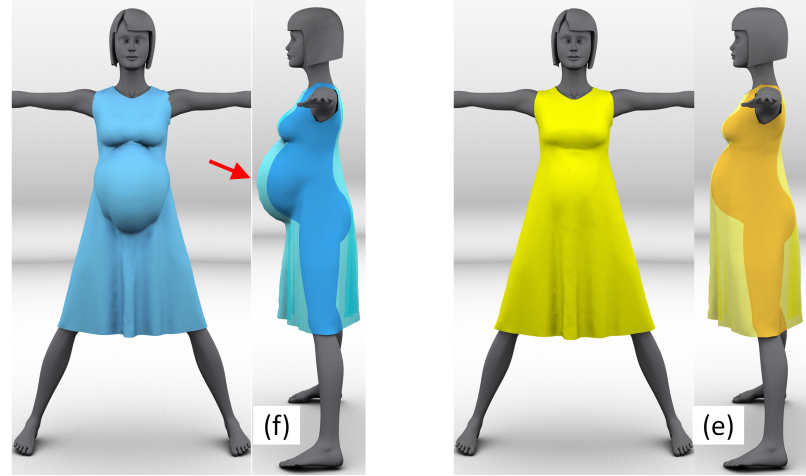

Figure 13: Top: A dress (a) transferred to a fatter model using skinning [Wang et al. 2005] (b) shows visible artifacts along silhouettes. On this dress $(c, d)$ our automatic method (yellow) generates slightly better results than the user assisted approach of Meng et al. [2012] (blue) (arrows point to undesirable artifacts). Bottom: When transferring a dress (Figure 12, top) to a more different pregnant model, using the indicated user-specified profiles (a) Meng et al. [2012] (blue) generate severe artifacts, resulting in a dress which is not physically plausible (floating around the belly). In contrast, our automatically generated output (yellow) reflects the input design. 
Harmon, D., Panozzo, D., Sorkine, O., And Zorin, D. 2011. Interference aware geometric modeling. ACM Transactions on Graphics 30, 6.

Igarashi, T., Moscovich, T., And Hughes, J. 2005. Asrigid-as-possible shape manipulation. ACM Transactions on Computer Graphics, Vol.24, No.3, ACM SIGGRAPH 2005, Los Angels, USA.

Kircher, S., AND GARLAND, M. 2006. Editing arbitrarily deforming surface animations. ACM Trans. Graph. 25 (July), 1098-1107.

Kraevoy, V., And Sheffer, A. 2004. Cross-parameterization and compatible remeshing of $3 \mathrm{~d}$ models. ACM Trans. Graph. 23, 861-869.

Kraevoy, V., Sheffer, A., Cohen-Or, D., And Shamir, A. 2008. Non-homogeneous resizing of complex models. ACM Trans. Graph, 111.

Mehra, R., Zhou, Q., Long, J., Sheffer, A., Gooch, A., AND MitRA, N. J. 2009. Abstraction of man-made shapes. ACM Transactions on Graphics 28, 5, \#137, 1-10.

Meng, Y., Wang, C. C., AND Jin, X. 2012. Flexible shape control for automatic resizing of apparel products. ComputerAided Design 44, 1, 68-76.

Moore, C. L., Mullet, K. K., And Young, M. B. P. 2001. Concepts of Pattern Grading: Techniques for Manual and Computer Grading. Fairchild Books And Visuals.

Nocedal, J., AND Wright, S. 2006. Numerical optimization. Springer series in operations research. Springer.

OPTITEX. www.optitex.com/en/products/main_modules/grade.

Protopsaltou, D., And Luible, C. 2002. A body and garment creation method for an internet-based virtual fitting room. Advances in Modeling, Animation and Rendering, J. Vince and R. Earnshaw, eds., Springer-Verlag, 105-122.

Robson, C., Maharik, R., Sheffer, A., And CARr, N. 2011. Context-aware garment modeling from sketches. Computers and Graphics (Proc. SMI 2011), 604-613.

ShefFer, A., LÉvy, B., MOgILnitsky, M., AND BoGOMYAKOV, A. 2005. Abf++: fast and robust angle based flattening. ACM Trans. Graph. 24 (April), 311-330.

Sheffer, A., Praun, E., AND Rose, K. 2006. Mesh parameterization methods and their applications. Found. Trends. Comput. Graph. Vis. 2, 105-171.

Sorkine, O., AND AleXA, M. 2007. As-rigid-as-possible surface modeling. Eurographics Symposium on Geometry Processing.

Sumner, R., And Popovic, J. 2004. Deformation transfer for triangle mesh. ACM Trans. Graph. 23, 3, 399-405.

Takayama, K., Schmidt, R., Singh, K., Igarashi, T., BoubeKeur, T., AND Sorkine, O. 2011. Geobrush: Interactive mesh geometry cloning. Computer Graphics Forum (proceedings of Eurographics) 30, 2, 613-622.

THREADS. www.threadsmagazine.com/item/4368/

making - sense - of - pattern - grading.

Turquin, E., Wither, J., Boissieux, L., Cani, M.-P., And HugHES, J. 2007. A sketch-based interface for clothing virtual characters. IEEE Computer Graphics \& Applications.
Umetani, N., Kaufman, D. M., Igarashi, T., And GrinSPUN, E. 2011. Sensitive couture for interactive garment editing and modeling. ACM Transactions on Graphics (SIGGRAPH 2011) 30, 4.

Volino, P., Cordier, F., and Magnenat-thalmann, N. 2005. From early virtual garment simulation to interactive fashion design. Computer-Aided Design 37, 593608.

Wang, C. C. L., Wang, Y., And Yuen, M. M. F. 2005. Design automation for customized apparel products. Comput. Aided Des. 37, 675-691.

WANG, C. C. L., C. Hui, K., AND M. Tong, K. 2007. Volume parameterization for design automation of customized free-form products. Submitted for IEEE Transactions on Automation Science and Engineering 4, 11-21. 